EPJ Web of Conferences 28, 12017 (2012)

DOI: $10.1051 /$ epjconf/20122812017

(C) Owned by the authors, published by EDP Sciences, 2012

\title{
Search for supersymmetry in events with a $Z$ boson, jets and missing energy
}

\author{
Marco - Andrea Buchmann ${ }^{1, \mathrm{a}}$ on behalf of the CMS Collaboration \\ Institute for Particle Physics, ETH Zürich, CH-8093 Zürich, Switzerland
}

\begin{abstract}
We present a search for Physics beyond the Standard Model (SM) in final states with a Z boson, jets and missing transverse energy, using a data sample collected in 2011 by the CMS detector at the Large Hadron Collider corresponding to an integrated luminosity of $2.1 \mathrm{fb}^{-1}$. This final state is predicted in several models of Physics beyond the SM, including supersymmetry. A novel analysis method is exploited, the Jet-Z Balance method, and a precise determination of the total SM background is obtained using a control sample from data. In the absence of any significant excess beyond the SM background, upper limits are set on simple models of supersymmetry, and further information is provided to allow confrontation of other models to these results.
\end{abstract}

We describe a search [1] for Physics beyond the Standard Model (BSM) in a sample of pp collisions collected by the Compact Muon Solenoid (CMS) detector [2] at the Large Hadron Collider (LHC), at a center-of-mass energy of $7 \mathrm{TeV}$. The size of the data sample corresponds to $2.1 \mathrm{fb}^{-1}$. We search for final states with a $\mathrm{Z}$ boson, jets and missing energy $\left(E_{T}^{\text {miss }}\right)$ where the $\mathrm{Z}$ boson decays to electron or muon pairs. This final state is a clean and distinct signature present in many models of BSM Physics, in particular supersymmetry (SUSY) [3,4].

The most significant Standard Model (SM) backgrounds in this final state are $Z+$ jets processes and $\bar{t} \bar{t}$ production. The former is the most challenging since it peaks in the mass distribution of the two leptons and can have apparent missing energy due to detector resolution and reconstruction effects.

The Jet-Z balance (JZB) method has been devised to predict the contribution from $Z+$ jets events [5]. We use the flavor symmetry of $\mathrm{t} \overline{\mathrm{t}}$ decays, as well as dilepton invariant mass side-band regions to predict the contribution from $t \bar{t}$ events.

We select events with at least three central jets with transverse momentum $p_{T}>30 \mathrm{GeV}$ and two opposite-sign leptons with $p_{T}>20 \mathrm{GeV}$.

The JZB variable is defined as the difference between the $p_{T}$ of the vectorial sum of all jets and the $p_{T}$ of the $\mathrm{Z}$ boson candidate:

$$
J Z B=\left|\sum_{\text {jets }} \mathbf{p}_{T}\right|-\left|\mathbf{p}_{T}^{(Z)}\right|
$$

The JZB variable has a strong discriminating power between instrumental MET events (which populate the left and the right side evenly) and real MET events, like signal events, which are shifted to the right. The JZB distribution is shown in Fig. 1.

A low-, a mid-, and a high-JZB search region are defined by requiring $\mathrm{JZB}>50 \mathrm{GeV}, \mathrm{JZB}>100 \mathrm{GeV}$, and $\mathrm{JZB}>150 \mathrm{GeV}$, respectively. These requirements efficiently

\footnotetext{
a e-mail: marco. andrea.buchmann@cern.ch
}



Fig. 1. JZB distribution in $Z+$ jets, $\overline{\mathrm{t}} \mathrm{t}$ and signal MC simulation scaled to $2.1 \mathrm{fb}^{-1}$, after preselection and dilepton invariant mass requirement. The signal distribution corresponds to the SUSY LM4 scenario.

suppress the $Z+$ jets background, leaving $\mathrm{t} \overline{\mathrm{t}}$ as the dominant background.

The signal region, denoted $\mathrm{JZB}_{\text {pos }}^{\mathrm{SFZP}}$, consists of sameflavor events in which the invariant mass of the two leptons is compatible with the $\mathrm{Z}$ mass hypothesis $\left(\left|m_{\ell \ell}-m_{Z}\right|<\right.$ $20 \mathrm{GeV}$ ) and $\mathrm{JZB}>0 \mathrm{GeV}$. Three control regions are used to predict the contribution of flavor-symmetric backgrounds (mainly coming from $\mathrm{t} \overline{\mathrm{t}}$ processes):

- opposite-flavor events compatible with the Z boson mass hypothesis $\left(\mathrm{JZB}^{\mathrm{OFZP}}\right)$

- opposite-flavor events in the side-band of the $\mathrm{Z}$ boson mass $\left(\mathrm{JZB}{ }^{\mathrm{OFSB}}\right)$ and

- same-flavor events in the same side-band region $\left(\mathrm{JZB}^{\mathrm{SFSB}}\right)$

The side-band region is defined as $55 \mathrm{GeV}<m_{\ell \ell}<70 \mathrm{GeVU}$ $112 \mathrm{GeV}<m_{\ell \ell}<160 \mathrm{GeV}$. 

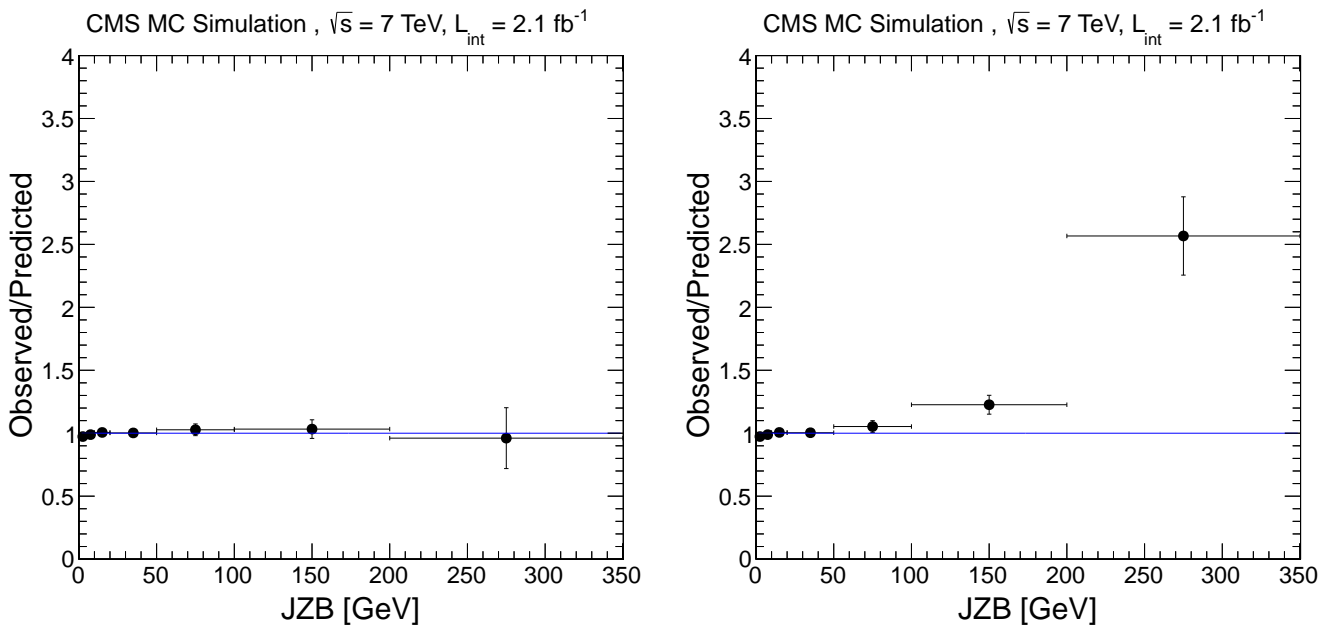

Fig. 2. Ratio between observed and predicted JZB distributions in Monte Carlo simulation, without (left) and with (right) the inclusion of signal Monte Carlo simulation. The errors correspond to the statistical uncertainty of the Monte Carlo simulation.
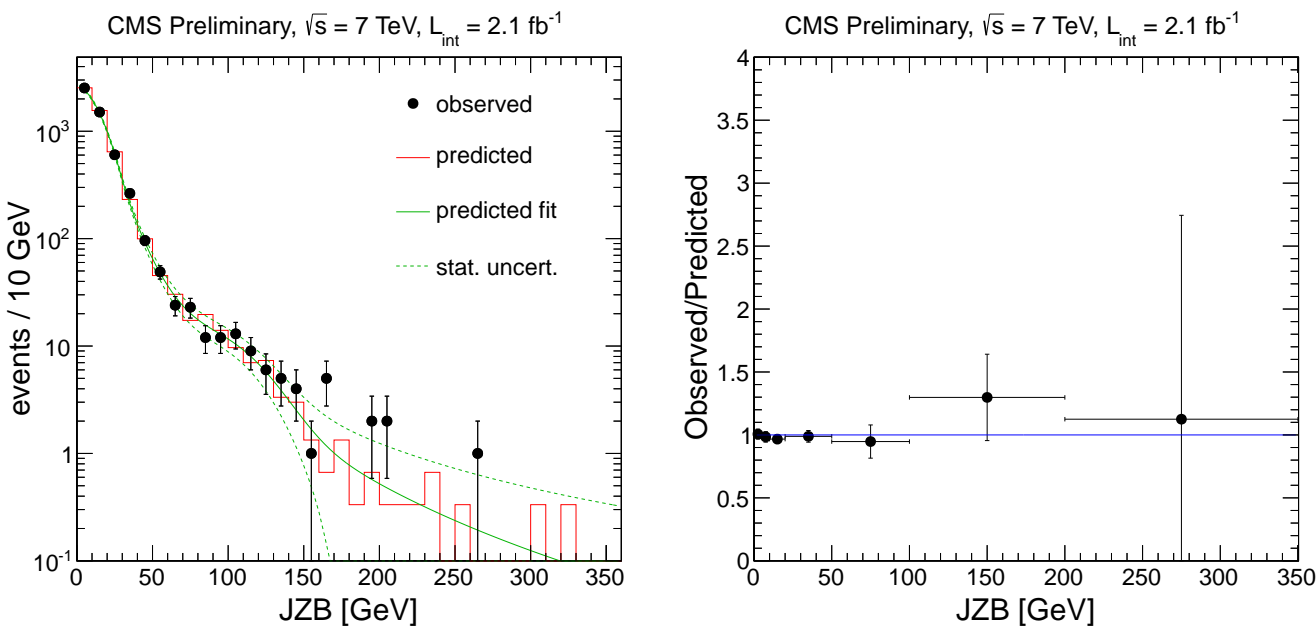

Fig. 3. Comparison between the predicted and observed JZB distributions in the search region. The predicted distribution has been fitted to display 1- $\sigma$ error bands. The right plot shows the ratio between the two distributions on the left.

The contribution from flavor-symmetric backgrounds is computed as the average of the three control regions, as they provide an independent estimate of the same background process. The contribution from the $Z+$ jets background is estimated from the $\mathrm{JZB}_{\text {neg }}^{\mathrm{SFP}}$ region after subtracting the contribution from flavor-symmetric backgrounds in this region. The total background prediction in the signal region is then computed as:

$$
\begin{aligned}
J Z B_{\text {bkgd }}^{\text {pred }}=\left|\mathrm{JZB}_{\text {neg }}^{\mathrm{SFZP}}\right| & +\frac{1}{3}\left|\mathrm{JZB}_{\text {pos }}^{\mathrm{OFZP}}\right|-\frac{1}{3}\left|\mathrm{JZB}_{\text {neg }}^{\mathrm{OFZP}}\right| \\
& +\frac{1}{3}\left|\mathrm{JZB}_{\text {pos }}^{\mathrm{SFSB}}\right|-\frac{1}{3}\left|\mathrm{JZB}_{\text {neg }}^{\mathrm{SFSB}}\right| \\
& +\frac{1}{3}\left|\mathrm{JZB}_{\text {pos }}^{\mathrm{OFSB}}\right|-\frac{1}{3}\left|\mathrm{JZB}_{\text {neg }}^{\mathrm{OFSB}}\right|
\end{aligned}
$$

We assign a systematic uncertainty of $25 \%$ to $\mathrm{JZB}_{\text {neg }}^{\mathrm{SFZP}}$ and $\mathrm{JZB}_{\text {pos }}^{\mathrm{SFZP}}$ and a systematic uncertainty of $50 \%$ to each of the estimates from the three control regions for flavorsymmetric backgrounds. The background estimation methods are validated in Monte Carlo simulation, in a mix- ture of all SM backgrounds, with and without the inclusion of signal Monte Carlo simulation. The ratio between observed and predicted distributions is shown in Fig. 2 for the two cases. There is very good agreement in the background-only hypothesis and good sensitivity to a possible signal.

The comparison between predicted and observed distributions in data is shown in Fig. 3. The observed and predicted yields in these regions are summarised in Tab. 1.

Table 1. Total number of events observed in the search regions, and corresponding background prediction.

\begin{tabular}{lcc}
\hline Region & Observed & Background prediction \\
\hline $\mathrm{JZB}>50 \mathrm{GeV}$ & 168 & $164 \pm 10($ stat $) \pm 42($ sys $)$ \\
$\mathrm{JZB}>100 \mathrm{GeV}$ & 48 & $37 \pm 4($ stat $) \pm 10($ sys $)$ \\
$\mathrm{JZB}>150 \mathrm{GeV}$ & 11 & $7.0 \pm 1.5($ stat $) \pm 2.1($ sys $)$ \\
\hline
\end{tabular}






Fig. 4. Simplified model for the production of two gluinos decaying into jets and a $\mathrm{Z}$ boson

In the absence of any significant excess, we set upper limits on the production cross-section of simplified models of SUSY (SMS). In the process considered in this study (shown in Fig. 4), two gluinos are generated, decaying to jets and a neutralino, which in turn decays to a $\mathrm{Z}$ boson and the lightest SUSY particle (LSP). The parameters of the model are the masses of the gluino $\left(m_{\tilde{g}}\right)$ and the $\operatorname{LSP}\left(m_{\tilde{\chi}_{1}^{0}}\right)$. The mass of the intermediate neutralino $\left(m_{\tilde{\chi}_{2}^{0}}\right)$ is fixed to the mean of the two other masses. Signal contamination is fully accounted for and the obtained limits are reliable.

The 95\% CL upper limits on the cross-section for the topology described above are shown in Fig. 5. The upper limits are computed using a frequentist $\mathrm{CL}_{\mathrm{S}}$ method [6]. In order to interpret these limits in terms of gluino pair production cross-section, we use a reference cross-section $\sigma_{\text {ref }}$ and draw the 95\% CL exclusion contours on $\frac{1}{3}, 1$ and 3 times $\sigma_{\text {ref. }}$. The reference cross-section corresponds to gluino pair production in the limit of infinitely heavy squarks, calculated at next to leading order using PROSPINO [7] and CTEQ6 [8] parton distribution functions.

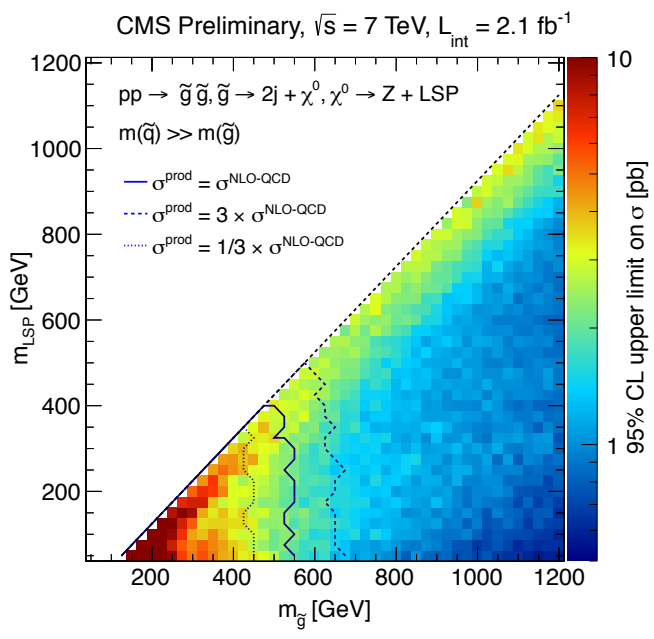

Fig. 5. 95\% CL upper limits on the cross-section of the inclusive $\mathrm{Z}$ boson decay mode in the $\left(m_{\tilde{q}}, m_{\mathrm{LSP}}\right)$ SMS parameter space. In each point, the best expected limit from the low-, mid-, and highJZB regions is used. The exclusion curves for reference crosssections are also shown in this plot.

In this simplified model, the JZB analysis is mostly sensitive to topologies in which the $\mathrm{Z}$ boson and the LSP have a momentum in the rest frame of the neutralino that is small with respect to the momentum of the neutralino [9]. In the considered set of simplified models, this topology is realized in the region where the difference between the
LSP mass and the gluino mass is small, as the JZB distribution for signal is very asymmetric and signal contamination has little impact on the sensitivity.

We also estimate upper limits in the context of two CMSSM benchmark scenarios, the LM4 and LM8 scenarios (see Tab. 2). LM4 (and LM8) are defined as $m_{0}=$ $210(500) \mathrm{GeV}, m_{1 / 2}=285(300) \mathrm{GeV}, \tan \beta=10, \operatorname{sign}(\mu)=$ + , and $A_{0}=0(-300) \mathrm{GeV}$, respectively. The LM4 scenario is excluded at $95 \%$ CL by our search.

Table 2. Observed upper limits on the cross section of LM4 and LM8 benchmark points for different cuts on JZB. The last column indicates the NLO cross-section of the two scenarios.

\begin{tabular}{lcccc}
\hline Scenario & $>50 \mathrm{GeV}$ & $>100 \mathrm{GeV}$ & $>150 \mathrm{GeV}$ & $\sigma$ \\
\hline LM4 & $7.4 \mathrm{pb}$ & $3.8 \mathrm{pb}$ & $1.9 \mathrm{pb}$ & $2.53 \mathrm{pb}$ \\
$\mathrm{LM} 8$ & $7.9 \mathrm{pb}$ & $4.2 \mathrm{pb}$ & $2.0 \mathrm{pb}$ & $1.03 \mathrm{pb}$ \\
\hline
\end{tabular}

We have presented a search for physics beyond the SM in $Z+$ jets $+E_{T}^{\text {miss }}$ events using a data sample corresponding to an integrated luminosity of $2.1 \mathrm{fb}^{-1}$. We do not observe any deviation between the observed number of events in the signal region and the predicted number of events from data control samples. In the absence of any hint of Physics beyond the SM, we interpreted our result using simplified models of SUSY.

\section{References}

1. CMS Collaboration, "Search for Physics Beyond the Standard Model in Z + Jets + MET events at the LHC". SUS-PAS-11-019 (2011)

2. CMS Collaboration, "The CMS experiment at the CERN LHC", JINST 3:S08004 (2008)

3. K.T. Matchev, and S.D. Thomas, Phys. Rev. D62, (2000) 077702 doi:10.1103/PhysRevD.62.077702

4. J.T. Ruderman and D. Shih, "General Neutralino NLSPs at the Early LHC”, arXiv:1103.6083

5. K. Theofilatos, "Supersymmetric particle detection techniques and electromagnetic calorimeter testbeam analysis with the CMS detector". PhD thesis, National Tech. University of Athens and N.C.S.R. Demokritos, 2009.

6. K. Nakamura et al. (Particle Data Group), J. Phys. G 37, (2010) 075021. doi:10.1088/0954-3899/37/7A/075021.

7. W. Beenakker, R. Hoepker, and M. Spira, "PROSPINO: A Program for the Production of Supersymmetric Particles in Next-to-leading Order QCD", 1996

8. J. Pumplin et al., "New generation of parton distributions with uncertainties from global QCD analysis", JHEP 07, (2002) 012

9. CMS Collaboration, "Search for Physics Beyond the Standard Model in Z + MET + Jets events at the LHC". SUS-PAS-10-010 (2010) 